\title{
PARALISIA SUPRANUCLEAR PROGRESSIVA
}

(SÍNDROME DE STEBI^MOHARDSON-OLSZBWSXI)

APRESENTAÇÃO DE CASO E REVISiÃo DA LITERATURA

PAULO CESAR TREVISOL-BITTENCOURT *

\begin{abstract}
RESUMO - Paralisia supranuclear progressiva (PSP) foi inicialmente identificada como entidade distinta por Richardson, Steele e Olszewski há cerca de 25 anos. Observações clínicas subsequentes não apenas têm confirmado mas, também acrescentaram novas informações aos relatos pioneiros. PSP pode ser descrita como transtorno neurológico gradualmente incapacitante com rigidez extrapiramidal, bradieinesia, dificuldade na marcha com quedas frequentes, paralisia pseudo-bulbar, demência e oftalmoplegia supranuclear característica. Incide em pessoas na meia idade ou idosas, sem distinção racial ou sexual. PSP é importante causa de parkinsonismo e sua etiologia permanece obscura. Neste registro é apresentado o caso de um paciente de Santa Catarina, exibindo inequívoca evidência clínica de PSP. Este é o primeiro caso descrito neste Estado onde, baseando-se em recentes premissas epidemiológicas, poderemos supor <a existência de pelo menos mais 50 pacientes com PSP. Em complementação, é apresentada revisão da literatura, com ênfase a aspectos clínicos e terapêuticos da PSP.
\end{abstract}

PALAVRAS-CHAVE: paralisia supranuclear progressiva, parkinsonismo, demência, epilepsia, flunarizina.

Progressiiwe supranuclear palsy: a case report and review of literature

SUMMARY - Progressive supranuclear palsy (PSP) was first recognized as a distinct syndrome by Richardson, Steele and Olszewski roughly a quarter century ago. Subsequent clinical experience has corroborated and enlarged their original observations. PSP has become familiar as a chronic progressive disorder with extrapyramidal rigity, bradykinesia, gait impairment, bulbar palsy, dementia and a characteristic supranuclear ophthalmoplegia. It is a significant cause of parkinsonism and its etiology remains obscure. The case of a patient from Santa Oatarina who presented definite clinical evidences of this syndrome is reported. This is the first description in this southern Brazilian State, where at least 50 more patients should exist, if we may extrapolate the prevalence rate of this condition in developed countries to this well developed area of Brazil. A review of the literature was undertaken with emphasis on recent clinical and therapeutic aspects of PSP.

KEY WORDS: progressive supranuclear palsy, parkinsonism, dementia, epilepsy, flunarizin.

Paralisia supranuclear progressiva (PSP) é expressão cunhada por Steele, Richardson e Olszewski para uma síndrome neurológica progressiva que se apresenta com manifestações oculares, motoras e mentais. Ainda que pacientes com quadros semelhantes tenham sido referidos anteriormente $2,5,18,33^{\wedge}$ PSP foi enunciada como entidade distinta pela primeira vez, em 196324 , subsequentemente, esses mesmos autores ofereceram descrição pormenorizada que permitiu sua individualização clínico-patológica definitiva ${ }^{28}$. Sua etiologia permanece ignorada e

* Disciplina de Neurologia do Departamento de Clínica Médica e Grupo de Pesquisas em Engenharia Biomédica, Universidade Federal de Santa Catarina (UFSC).

Dr. Paulo Cesar Trevisol Bittencourt - Disciplina de Neurologia, Departamento de Clínica Médica - Hospital Universitário, UFSC - 88049 Florianópolis SC - Brasil. 
a maior parte dos pacientes afetados estarão entre os 50 e 70 anos. PSP não tem predileção racial e, apesar dos primeiros estudos apontarem maior preponderância em homens, dados recentes sugerem não haver diferença sexual significante. Possui prevalência baixa, com estimativa de 14 pacientes para cada milhão de habitantes e incidência anual de aproximadamente três casos na mesma população 9. O diagnóstico desta síndrome deveria ser considerado quando pacientes apresentarem sinais e sintomas de: rigidez sem tremor, demência, paralisia pseudobulbar ou distonia axial em extensão, coexistindo oftalmoplegia 2». Pacientes com PSP são frequentemente diagnosticados como tendo "Parkinson atípico"; aliás, é reconhecido que algo em torno de $4 \%$ dos pacientes, encaminhados a uma clínica neurológica com possível doença de Parkinson, preencherão todos os critérios para o diagnóstico de PSP. Dificuldades em diagnosticá-la corretamente decorrem não somente do seu insuficiente conhecimento entre os médicos mas, também, porque os sinais peculiares frequentemente só se tornam evidentes tar* diamente. O diagnóstico desta condição permanece sendo essencialmente clínico, ainda que a tomografia computadorizada (TC) e a ressonância nuclear magnética (RNM) possam fornecer informações corroborativas em alguns casos. Na prática, tais exames se prestam mais para a exclusão de algumas causas capazes de mimetizar PSP, antes que para sua confirmação diagnostica. Ao contrário da doença de Parkinson idiopatica, a PSP tem prognóstico sombrio, com duração desde os primeiros sintomas até a morte de 4 a 6 anos e, em média, sobrevida de 1,8 anos após o diagnóstiso, sendo broncopneumonia a causa mortis usual 1 . Drogas tentadas até o momento, quando muito, apresentam benefício apenas transitório. As alterações neuropatológicas marcantes são degenerações neurofibrilares e grânulo-vacuolares, perda de células nervosas e gliose. Estes achados característicos são frequentes em núcleos do tronco cerebral, diencéfalo e cerebelo; entretanto, mais recentemente, alterações típicas foram descritas comprometendo também a medula espinhal is.

Neste registro é apresentado o primeiro caso autóctone de PSP em Santa Catarina. Paralelamente, é feita revisão da literatura, com ênfase a aspectos clínicos e terapêuticos.

\section{RELATO DO CASO}

AS, um homem branco, de 65 anos de idade, da raça ariana, foi encaminhado para avaliação neurológica por estar apresentando sintomas de «provável Parkinson com esclerose cerebral». Seus sintomas haviam iniciado aproximadamente 4 anos antes, com deterioração gradual desde então. Ele tinha sido um metódico motorista de caminhão por quase 30 anos, não tendo história de qualquer enfermidade importante no passado. Foi tabagista por muitos anos, interrompendo o hábito quando os sintomas iniciaram e não havia antecedente de etilismo. Sua história familiar era negativa para doenças neurológicas. Segundo sua esposa seus problemas iniciaram com «quedas frequentes e inexplicáveis», fato que levou ao questionamento de déficit visual como causa; entretanto, o uso de lentes corretoras não melhorou sua performance motora, com «tropeções» persistindo quase diariamente. Nessas quedas havia tendência para «cair de costas». Concomitantemente, seus familiares passaram a observar também mudança na voz e comportamento. Um filho ilustrou a anamnense com a seguinte observação: «...o pai começou a ficar com a voz diferente e o raciocínio lento há muitos anos, mas nos últimos dois anos ele está completamente caduco; atualmente, quando muito, ele responde as perguntas simples que lhe fazemos emitindo sons ininteligíveis e ultimamente anda se engasgando com comida que nem uma criança...». Um diagnóstico de «uma forma atípica da doença de Parkinson» foi feita anteriormente; contudo, tratamento apropriado para esta condição durante os últimos três anos não resultou em qualquer benefício, não obstante os vários esquemas anti-parkinsonianos experimentados. Apesar disso, vinha utilizando cronicamente levodopa-j-carbidopa e biperideno, associados com ergocristina e flunarizina. Seu exame clínico geral evidenciava paciente de compleição física forte e aparentemente saudável, tendo hiperemia conjuntival bilateral como única anormalidade. Todavia, uma rica semiologia neurológica podia ser constatada. Tinha face «em máscara» e voz lenta, baixa e disártrica. Déficits acentuados foram vistos nos movimentos oculares, com alterações bilaterais sendo observadas. Havia importante limitação dos movimentos oculares conjugados, estando os movimentos verticais em qualquer sentido significativamente mais comprometidos que os laterais. A manobra dos «olhos de boneca» evidenciava que os reflexos vestíbulo-oculares estavam preservados. Um teste de fluência verbal foi feito e o paciente, durante 90 segundos, não conseguiu nomear mais que três palavras iniciando com S. Pobre desempenho na capacidade de abstração e em cálculos simples foi também notado, além de deficiência nas memórias recente e remota - aquela mais 


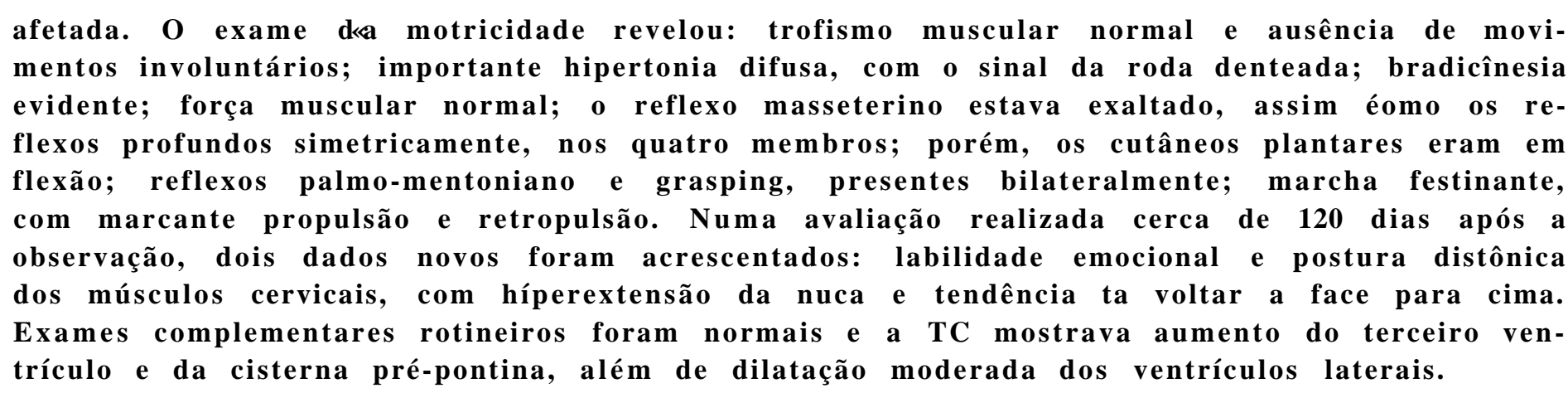

\section{COMENTÁRIOS}

O quadro clínico exibido pelo paciente AS - com a combinação de rigidez sem tremor, bradicinesia, demência, distonia, sinais pseudobulbäres e oftalmoplegia tipo supranuclear - preenche todos os critérios estabelecidos por Steele e col. para o diagnóstico de PSP 24,28,29. contudo, como o paciente estava usando cronicamente flunarizina, medicação com capacidade ímpar de produzir no usuário parkinsonismo e depressão ${ }^{31}$, nossa primeira atitude foi suprimi-la. Entretanto, reavaliações periódicas sucessivas mostraram que, apesar de melhora subjetiva, os sinais de parkinsonismo permaneceram inalterados.

Apesar de não ter a PSP padrão definido de apresentação, frequentemente distúrbios da marcha, como os apresentados por este paciente, serão sua manifestação inicial. Geralmente, pacientes (e familiares) tendem a referir "tropeções" frequentes e inexplicáveis. Aliás, revisões recentes sobre esta condição têm salientado como sintomas iniciais mais comuns: instabilidade na marcha, com quedas frequentes devidas a equilíbrio deficiente; distúrbios visuais; alteração na fala e evidências de deterioração intelectual 17. Todos esses sintomas foram apresentados por AS, desde o início do quadro.

A diminuição dos movimentos oculares voluntários, especialmente os verticais, é usualmente sinal precoce que resulta em queixas visuais. O caráter supranuclear e não paralítico desta alteração pode ser demonstrado pela preservação completa dos movimentos reflexos óculo-cefálicos, conforme evidenciado neste paciente. Entretanto, apesar de amplamente difundido e aceito, que "todos os aspectos da função oculomotora são quantitativamente anormais em pacientes com PSP quando um diagnóstico clínico é possível" 6, o diagnóstico desta condição deveria ser considerado mesmo na ausência das clássicas manifestações oculares pois, em alguns casos, estas alterações típicas poderão surgir tardiamente ou, até mais raramente, não apareceremia. Além disso, adicionando confusão diagnostica, pacientes com típica doença de Parkinson, poderão ocasionalmente apresentar oftalmoplegia supranuclear transitória. Nestes pacientes, quadros infecciosos sistêmicos devem ser pesquisados, pois poderão ser a causa!2. Por putro lado, deve-se ter em mente que paralisias supranucleares do olhar conjugado podem ter outras etiologias que PSP e, pelo fato de haver para algumas delas tratamento eficaz, a exclusão destas possibilidades é necessária 21,23.

Sob um ângulo neuropsiquiátrico, nosso paciente apresenta alterações sugestivas de demência subcortical, síndrome clínica que é caracterizada por lentificação mental com prejuízo da cognição, déficit de memória, apatia e depressão Demência subcortical foi reconhecida primeiramente em pacientes com PSP e coréia de Huntington. Entretanto, essa condição também tem sido aventada para explicar a deterioração intelectual que pode estar associada às doenças de Parkinson e de Wilson, degenerações espinocerebelares, calcificação idiopática dos gânglios da base, estado lacunar e a demência associada a depressão. Pacientes que manifestam demência subcortical têm alterações patológicas envolvendo primariamente o tálamo, gânglios basais e núcleos do tronco cerebral relacionados a essas estruturas, com pouco, se algum, envolvimento do córtex cerebral. As características clínicas da demência subcortical diferem daquelas vistas na demência tipo Alzheimer, em que o proeminente acometimento do córtex leva ao surgimento de afasia, agnosia e apraxia.

As semelhanças observadas entre as anormalidades neuropsicológicas vistas na PSP e nas síndromes do lobo frontal, juntamente com a falta de envolvi- 
mento cortical, sugerem que a demência da PSP poderia ser explicada pela desativação do córtex frontal por lesões subcorticais ${ }^{4} . \mathbf{3 0}$. Na verdade, recentes estudos realizados com tomografia por emissão de positrons reforçam esta hipótese, por demonstrarem que, apesar de anatomicamente íntegros, os lobos frontais são hipofuncionantes em pacientes com PSP 10. Por outro lado, deve-se ter em mente que sintomas psiquiatricos podem preceder o início dos déficits neurológicos ou mascará-los; desta maneira, a possibilidade de PSP também deveria ser considerada no diagnóstico diferencial das síndromes psiquiátricas de início tardio 26,32.

Distonias de membros isolados ou de região cervical, geralmente em extensão, são extremamente frequentes em pacientes com PSP e, não raro, serão sua primeira manifestação. Nosso paciente a exibia em região cervical. Devido à sua constância, a suposição diagnostica de PSP deveria ser feita naqueles pacientes em idade pré-senil ou senil que apresentem distonia não relacionada às inúmeras drogas capazes de desencadeá-la.

Os sinais e sintomas de paralisia pseudobulbar, como exteriorizados pelo nosso paciente, são vistos na maioria dos casos de PSP, durante sua evolução. Eventualmente, poderão ser os achados iniciais. Contudo, é oportuno recordar que paralisia pseudobulbar, assim como todos os sinais cardinais de PSP, poderão ser causados por múltiplos infartos cerebrais (MIF) e quadros semelhantes já têm sido relatados 7. A história prévia de acidentes vasculares, níveis tensionais elevados e outros fatores de risco para doença vascular cerebral, associados com a presença de déficits motores, apontam em direção ao diagnóstico de MIF; todavia, nesta situação, TC ou RNM serão imprescindíveis para um diagnóstico correto.

A despeito de não termos observado neste paciente a incidência elevada de crises epilépticas em pacientes com PSP, muito além do que poderia ser esperado por acaso, é aspecto interessante e intrigante 9 . Em publicação recente, Duvoisin e col. 9 encontraram a expressiva taxa de $11,2 \%$, dos seus pacientes com crises epilépticas. Desde que pacientes com típica doença de Parkinson raramente apresentam crises epilépticas", a ocorrência deste sintoma em pacientes com parkinsonismo será altamente sugestiva da possibilidade de PSP. Postula-se um envolvimento do hipocampo pelo processo degenerativo, para justificar tal susceptibilidade aumentada 20. Nestes pacientes, anormalidades eletrográficas, quando presentes, serão inespecíficas.

Exames complementares disponíveis no momento não são úteis para fins diagnósticos de PSP, embora a TC possa oferecer informações altamente sugestivas em alguns casos 25. Entretanto, como as lesões nesta síndrome são fundamentalmente infratentoriais, a RNM é o exame capaz de poder oferecer mais subsídios sobre esta condição nos dias atuais. Assim, tais estudos, quando indicados, deveriam ser feitos com o intuito de excluir causas capazes de mimetizar PSP e, nunca, com propósitos diagnósticos. Por outro lado, um criterioso "sereening" oncológico estaria indicado nestes pacientes, pois há relato de ser esta condição agravada por neoplasia primitiva de pulmão, por mecanismo paraneoplásico 15.

Enfocando aspectos terapêuticos, deve-se frisar inicialmente a inexistência de tratamento satisfatório para PSP. Apesar do relato anedotário de melhora marcante mas transitória com corticóide $n$, todos os ensaios realizados com diversas drogas até o presente tiveram resultados frustrantes. Há relatos de resposta fugaz ao uso de drogas dopaminérgicas pré-sinápticas, como levodopa e amantadina; entretanto, o número de pacientes beneficiados é reduzido. Recentes publicações sugerem que agonistas dopaminérgieos, como bromocriptina e pergolide por exemplo, poderiam trazer melhores resultados 34; todavia, os resultados obtidos com estas drogas se não são decepcionantes, também estão longe de qualquer ideal. Amitriptilina, um antidepressivo tricíelico, tem exibido alguma eficácia; porém o benefício tem se revelado pequeno e fugaz 19. Metisergida, um bloqueador serotonínico com potencial tóxico não desprezível!, foi tentada exaustivamente no passado, com resultados estimulantes para uns ${ }^{22}$ e não convincentes para outros 8. Contudo, desde que esta droga pode exercer ação positiva em poucos pacientes, particularmente naqueles com sintomas bulbares, um curto ensaio em pacientes com disfagia deveria ser considerado, pois as complicações de um curto ensaio serão mínimas e, naqueles pacientes responsivos, os riscos de toxicidade a longo prazo serão menores que aqueles da aspiração a que estão expostos 
Surpreendentemente, um promissor inibidor seletivo da MAOB (Deprenyl) ainda não foi testado em pacientes com PSP. Deprenyl é, pelo menos do ponto de vista teórico, uma alternativa atraente ${ }^{2}$ ?. Apesar da inexistência de terapêutica medicamentosa eficiente nos dias atuais, deve ser reconhecido que técnicas de reabi* litação adequadas poderão melhorar o status funcional desses pacientes por bom período $^{14}$. Pacientes e familiares devem ser encorajados neste sentido.

Finalmente, considerando que somente $15 \%$ dos pacientes com doença de Parkinson não responderão adequadamente a levodopa 3, quando um paciente com parkinsonismo não mostrar melhora significativa diante do tratamento standard, várias questões devem ser formuladas. Entretanto, a primeira deverá ser: o diagnóstico está correto? Apesar de ser a doença de Parkinson a causa mais frequente, o parkinsonismo tem múltiplas possibilidades etiológicas e o diagnóstico de PSP deveria ser sempre considerado naqueles pacientes apresentando quedas rotineiras com sinais e sintomas de rigidez sem tremor, demência, paralisia pseudobulbar ou distonia axial, combinados ou não a oftalmoplegia. Aliás, alterações dos movimentos oculares deveriam ser sistematicamente pesquisados em todos os pacientes exibindo essas alterações. Em decorrência disso, redução expressiva no número de pacientes com o diagnostico de doença de Parkinson atípica poderia ser esperada.

Agradecimentos - $O$ autor expressa sua gratidão ao apoio material da National Socíety for Epilepsy do Reino Unido; ao inestimável estímulo do amigo Ley Sander; ao Dr. Li Shih Man, por ter referido o paciente; aos Drs. Reiuato Garcia Ojeda e Monique von Hertwig, pela valiosa ajuda na redação deste artigo.

\section{REFERÊNCIAS}

1. Bernick C. Methysergide-induced akathisia. Clin Neuropharmacol 1988, 11:87-89.

2. Brusa A. Dégénérescence plurisystematisée du neuraxe de caractère sporadique, à debut tardif et évolution sutmiguë. Rev Neurol 1961, 104:412-429.

3. Bruns R. Difficulties in long term management of Parkinson's disease. Aust Fam Psysician 1984, 13:14-16.

4. Cambier J, Masson M, Viader F. Limodin J, Strube A. Le syndrome frontal de la paralysie supranucléaire progressive. Rev Neurol 1985, 141:528-536.

5. Chavany JA, van Bogaert L, Godlewski S. Sur un syndrome de rigidité à predominance -axiale avec perturbation des automatismes oculo-palpebraux d'origine encephalitique. Presse Med 1957, 59:958-962.

6. Daroff RB. Progressive supranuclear palsy: a brief personalized history. Yale J Biol Med 1987, 60:119-122.

7. Dubinsky RM, Jankovic J. Progressive supranuclear palsy and a multi-infarct state. Neurology 1987, 37:570-576.

8. Duncombe AS, Lees AJ. Methysergide in progressive supranuclear palsy. Neurology 1985, 35:936-937.

9. Duvoisin RC, Golbe LI, Lepore FE. Progressive supranuclear palsy. Can J Neurol Sei 1987, 14:547-554.

10. Goffinet AM, De Voider AG, Gillain C, Rectem D, Bol A, Michel C, Cogneau M, Lobar D, Laterre C. Positron tomography demonstrates frontal lobe hypometabolism in progressive supranuclear palsy. Ann Neurol 1989, 25:131-139.

11. Gomori AJ, Sima AAF. An atypical case of progressive supranuclear palsy. Can J Neurol Sei 1984, 11:4S-52.

12. Guiloff RJ, George RJ, Marsden CD. Reversible supranuclear ophthalmoplegia associated with parkinsonism. J Neurol Neuro sur g- Psychiatry 1980, 43:552-554.

13. Imai H, Narabayashi $H$, Safoata E. «Pure akinesia» and the later added supranuclear ophthalmoplegia. Adv Neurol 1987, 45:207-212.

14. Izzo KL, Dilorenzo $P$, Roth A. Rehabilitation in progressive supranuclear palsy: case report. Arch Phys Med Rehab 1986, 67:473-476.

15. Jankovic J. Progressive supranuclear palsy: paraneoplastic effect of bronchial carcinoma. Neurology 1985, 35:446-447.

16. Kato T, Hirano A, Weinberg MN, Jacobs AK. Spinal cord lesions in progressive supranuclear palsy: some new observations. Acta Neuropathol 1986, 17:11-14.

17. Mäher ER, Lees AJ. The clinical features and natural history of the Steele-RichardsonOlszewski syndrome. Neurology 1983, 13:273-278. 
IS. Neumann MA. Heterogenous system degeneration with particular involvement of reticular substance: correlation with neurologic disorders and the concept of Creutzfeld-Jacob disease. J Neuropath Exp Neurol 1961, 20:316.

19. Newman GC. Treatment of progressive supranuclear palsy with tricyclic antidepressants. Neurology 1985, 35:1189-1193.

20. Nygaard TG, Duvoisin RC, Manocha M, Chokroverty S. Seizures in progressive supranuclear palsy. Neurology 1989, 39:138-140.

21. Page NGR, Lean JS, Sanders MIX Vertical supranuclear gaze palsy with secondary syphilis. J Neurol Neurosurg Psychiatry 1982, 45:86-88.

22. Rafal RD, Grimm RJ. Progressive supranuclear pallsy: functional analysis of the response to methysergide and antiparkinsonian agents. Neurology 1981, 31:1507-1518.

23. Ramachandran T, Kim RC, Culebras A. Progressive supranuclear palsy and falcotentorial meningioma. Arch Neurol 1982, 39:68.

24. Richardson JC, Steele J, Olszweski J. Supranuclear ophthalmoplegia, pseudobulbar palsy, nuchal dystonia and dementia: a dlinical report on eight cases of heterogenous system degenerations Trans Am Neurol Assoc 1963, 88:25-29.

25. Saitoh H, Yoshii F, Shinotoara Y. Computed tomographic findings in progressive supranuclear palsy. Neuroradiology 1987, 29:168-171.

26. Schneider LS, Chui HC, Progressive supranuclear palsy manifesting with depressive features. J Am Geriatr Soc 1986, 34:663-665.

27. Sonsalla PK, Golbe LI. Deprenyl as prophylaxis against Parkinson's disease. Clin Neuropharmacol 1988, 11:387-400.

28. Steele J, Richardson JC, Olszweski J. Progressive supranuclear palsy. Arch Neurol 1964, 10:333-359.

29. Steele J. Progressive supranuclear palsy. Brain 1972, 95:693-704.

30. Strub RL. Frontal lobe syndrome in a patient with bilateral globus pallidus lesions. Arch Neurol 1989, 46:1024-1027.

31. Trevisol-Bittencourt PC. Flunarizina induzindo> parkinsonismo. Arq Cat Med 1990, 19:81-84.

32. Trzepacz PT, Murcko AC, Gillespie MP. Progressive supranuclear palsy misdiagnosed as schizophrenia. J Nerv Ment Pis 1985, 173:377-378.

33. Verhaart WJC. Degeneration of the brain stem and the cerebellum: an example of heterogenous systemic degeneration of the central nervous system. J Neuropath Exp Neurol 1958, 17:382-391.

34. Watts RL, Krieg MM, Freeman A, Mandir A, Iuvone PM. Progressive supranuclear palsy: clinical, physiological, radiographic and biochemical assessment. Abstracts of XIV World Congress of Neurology. October 22-27, New Delhi, 1989. 